\title{
POISSON BRACKETS DETERMINED BY JACOBIANS
}

\author{
Jaehyun Ahn*, Sei-Qwon OH**, And Sujin ParK***
}

\begin{abstract}
Fix $n-2$ elements $h_{1}, \cdots, h_{n-2}$ of the quotient field $B$ of the polynomial algebra $\mathbb{C}\left[x_{1}, x_{2}, \cdots, x_{n}\right]$. It is proved that $B$ is a Poisson algebra with Poisson bracket defined by $\{f, g\}=$ $\operatorname{det}\left(\operatorname{Jac}\left(f, g, h_{1}, \cdots, h_{n-2}\right)\right)$ for any $f, g \in B$, where $\operatorname{det}(\mathrm{Jac})$ is the determinant of a Jacobian matrix.
\end{abstract}

In [1], Jordan and the author studied Poisson brackets on the polynomial algebra $\mathbb{C}[x, y, z]$ in three indeterminates $x, y, z$ determined by Jacobians. In particular, for an arbitrary rational function $s / t \in \mathbb{C}(x, y, z)$, they analyzed the Poisson bracket determined by the formula

$$
(\{x, y\},\{y, z\},\{z, x\})=t^{2} \nabla(s / t), \quad s / t \in \mathbb{C}(x, y, z),
$$

where $\nabla=\left(\frac{\partial}{\partial x}, \frac{\partial}{\partial y}, \frac{\partial}{\partial z}\right)$ is the gradient. The general rule is that, for $f, g \in \mathbb{C}[x, y, z]$,

$$
\{f, g\}=t^{2} \operatorname{det}(\operatorname{Jac}(f, g, s / t)),
$$

where $\operatorname{det}(\operatorname{Jac}(f, g, s / t))$ denotes the determinant of the Jacobian matrix of $(f, g, s / t)$.

The purpose of this paper is to generalize the bracket (1) to the general polynomial algebra $A:=\mathbb{C}\left[x_{1}, x_{2}, \cdots, x_{n}\right], n \geq 3$. For fixed $n-2$ elements $h_{1}, \cdots, h_{n-2}$ of the quotient field $B$ of $A$, the fact that, for any $f, g \in B$, the bracket defined by

$$
\{f, g\}=\operatorname{det}\left(\operatorname{Jac}\left(f, g, h_{1}, \cdots, h_{n-2}\right)\right)
$$

is a Poisson bracket is proved in [4] and [2, Theorem 1.4]. But the proof of [4] is not clear and that of [2] uses the Plücker relation and special derivations induced by the $n$-Jacobi identity in [3]. Here we prove by using only elementary algebraic theories that (2) is a Poisson bracket on $B$. Fix $s_{1}, t_{1}, \cdots, s_{n-2}, t_{n-2} \in A$ such that $s_{i}$ and $t_{i} \neq 0$ are coprime

Received January 24, 2013; Accepted April 04, 2013.

2010 Mathematics Subject Classification: Primary 17B63; Secondary 16 S36.

Key words and phrases: Poisson bracket, Jacobian matrix, polynomial algebra.

Correspondence should be addressed to Sei-Qwon Oh, sqoh@cnu.ac.kr.

**Supported by a research fund of Chungnam National University. 
for each $i=1,2, \cdots, n-2$. Next, as a corollary, we obtain that, for all $f, g \in A$,

(3) $\{f, g\}=\left(t_{1} \cdots t_{n-2}\right)^{2} \operatorname{det}\left(\operatorname{Jac}\left(f, g, s_{1} / t_{1}, s_{2} / t_{2}, \cdots, s_{n-2} / t_{n-2}\right)\right)$

is a Poisson bracket in $A$, which is a generalization of (1). The presence of the factor $\left(t_{1} \cdots t_{n-2}\right)^{2}$ ensures that this restricts to a Poisson bracket on $A$.

Throughout the paper, $A$ and $B$ denote the algebra $\mathbb{C}\left[x_{1}, x_{2}, \cdots, x_{n}\right]$ with $n \geq 3$ and the quotient field of $A$ respectively, as above.

Notation 1. Let $\mathcal{F}=\left(\varphi^{i j}\right)$ be an $(n-2) \times n$-matrix with entries $\varphi^{i j} \in B$.

(a) For any $i, j=1, \cdots, n$, denote by $\mathcal{F}_{i j}$ the determinant of the $n \times n$-matrix $\left(\begin{array}{c}e_{i} \\ e_{j} \\ \mathcal{F}\end{array}\right)$, where $\left\{e_{i}\right\}_{i=1}^{n}$ is the standard basis of $B^{n}$.

(b) For $z \in B, \nabla z$ denotes the row vector of $B^{n}$

$$
\nabla z=\left(\frac{\partial z}{\partial x_{1}}, \frac{\partial z}{\partial x_{2}}, \cdots, \frac{\partial z}{\partial x_{n}}\right)=\frac{\partial z}{\partial x_{1}} e_{1}+\frac{\partial z}{\partial x_{2}} e_{2}+\cdots+\frac{\partial z}{\partial x_{n}} e_{n} .
$$

Lemma 2. For all $i, j=1, \cdots, n, \mathcal{F}_{i j}=-\mathcal{F}_{j i}$. In particular, $\mathcal{F}_{i i}=0$.

Proof. It is clear from the elementary linear algebra.

For any $(n-2) \times n$-matrix $\mathcal{F}$ in Notation 1 and for any $f, g \in B$, set

$$
\{f, g\}^{\mathcal{F}}=\left|\begin{array}{c}
\nabla f \\
\nabla g \\
\mathcal{F}
\end{array}\right| \in B
$$

Then the bilinear product $\{\cdot, \cdot\}^{\mathcal{F}}$ in (4) is antisymmetric and satisfies the Leibniz rule. Thus the algebra $B$ with the bilinear product $\{\cdot, \cdot\}^{\mathcal{F}}$ becomes a Poisson algebra if and only if $\{\cdot, \cdot\}^{\mathcal{F}}$ satisfies the Jacobi identity. In such case, $\{\cdot, \cdot\}^{\mathcal{F}}$ is said to be a Poisson bracket in $B$.

Notation 3. For $a, b, c \in B$, let

$$
J_{\mathcal{F}}(a, b, c)=\left\{\{a, b\}^{\mathcal{F}}, c\right\}^{\mathcal{F}}+\left\{\{b, c\}^{\mathcal{F}}, a\right\}^{\mathcal{F}}+\left\{\{c, a\}^{\mathcal{F}}, b\right\}^{\mathcal{F}} .
$$

Thus $a, b$ and $c$ satisfy the Jacobi identity for $\{\cdot, \cdot\}^{\mathcal{F}}$ if and only if $J_{\mathcal{F}}(a, b, c)=0$.

Note that any derivation on an algebra is determined by values of generators. 
Proposition 4. [1, Proposition 1.14] The algebra $B$ is a Poisson algebra under $\{\cdot, \cdot\}^{\mathcal{F}}$ if and only if $J_{\mathcal{F}}\left(x_{i}, x_{j}, x_{k}\right)=0$ for any $1 \leq i, j, k \leq$ $n$.

Lemma 5. Let $1 \leq i, j, k \leq n$. Then $J_{\mathcal{F}}\left(x_{i}, x_{j}, x_{k}\right)=0$ if and only if

$$
\sum_{\ell=1}^{n}\left[\frac{\partial \mathcal{F}_{i j}}{\partial x_{\ell}} \mathcal{F}_{\ell k}+\frac{\partial \mathcal{F}_{j k}}{\partial x_{\ell}} \mathcal{F}_{\ell i}+\frac{\partial \mathcal{F}_{k i}}{\partial x_{\ell}} \mathcal{F}_{\ell j}\right]=0 .
$$

Proof. If $i=j, i=k$ or $j=k$ then we have $J_{\mathcal{F}}\left(x_{i}, x_{j}, x_{k}\right)=0$ since $\{\cdot, \cdot\}^{\mathcal{F}}$ is antisymmetric, and (5) holds by Lemma 2. Hence we may assume that $i<j<k$. Observe that

$$
\begin{aligned}
J_{\mathcal{F}}\left(x_{i}, x_{j}, x_{k}\right) & =\left|\begin{array}{c}
\nabla \mid e_{i} \\
e_{j} \\
\mathcal{F}
\end{array}\right|+|\nabla| \begin{array}{c}
e_{j} \\
e_{k} \\
\mathcal{F}
\end{array}|| \begin{array}{c}
\nabla \\
\mathcal{F}
\end{array}|+| \begin{array}{c}
e_{k} \\
e_{i} \\
\mathcal{F}
\end{array}|| \begin{array}{c}
\mathcal{F} \\
e_{j} \\
\mathcal{F}
\end{array} \mid \\
& =\left|\begin{array}{c}
\sum_{\ell=1}^{n} \frac{\partial \mathcal{F}_{i j}}{\partial x_{\ell}} e_{\ell} \\
e_{k} \\
\mathcal{F}
\end{array}\right|+\left|\begin{array}{c}
\sum_{\ell=1}^{n} \frac{\partial \mathcal{F}_{j k}}{\partial x_{\ell}} e_{\ell} \\
e_{i} \\
\mathcal{F}
\end{array}\right|+\left|\begin{array}{c}
\sum_{\ell=1}^{n} \frac{\partial \mathcal{F}_{k i}}{\partial x_{\ell}} e_{\ell} \\
e_{j} \\
\mathcal{F}
\end{array}\right| \\
& =\sum_{\ell=1}^{n}\left[\frac{\partial \mathcal{F}_{i j}}{\partial x_{\ell}} \mathcal{F}_{\ell k}+\frac{\partial \mathcal{F}_{j k}}{\partial x_{\ell}} \mathcal{F}_{\ell i}+\frac{\partial \mathcal{F}_{k i}}{\partial x_{\ell}} \mathcal{F}_{\ell j}\right] .
\end{aligned}
$$

Hence the result holds.

Lemma 6. For any $1 \leq i, j, k, \ell \leq n$,

$$
\mathcal{F}_{i j} \mathcal{F}_{\ell k}+\mathcal{F}_{j k} \mathcal{F}_{\ell i}+\mathcal{F}_{k i} \mathcal{F}_{\ell j}=0
$$

Proof. If any two indices among $i, j, k, \ell$ are equal, say $\ell=i$, then

$$
\mathcal{F}_{i j} \mathcal{F}_{\ell k}+\mathcal{F}_{j k} \mathcal{F}_{\ell i}+\mathcal{F}_{k i} \mathcal{F}_{\ell j}=\mathcal{F}_{i j} \mathcal{F}_{i k}+\mathcal{F}_{j k} \mathcal{F}_{i i}+\mathcal{F}_{k i} \mathcal{F}_{i j}=0
$$

since $\mathcal{F}_{i i}=0$ and $\mathcal{F}_{k i}=-\mathcal{F}_{i k}$, and thus (6) holds in this case. So we may assume that $n \geq 4$ and that all $i, j, k, \ell$ are distinct. For any $p, q$ such that $1 \leq p, q \leq n$ and $p \neq q$, denote by $X_{p q}$ the set of all bijective maps from $\{1, \cdots, n-2\}$ onto $\{1, \cdots, n\} \backslash\{p, q\}$ and, for $p=1, \cdots, n-2, q=$ $1, \cdots, n$, denote by $z_{p q}$ the $(p, q)$-entry of $\mathcal{F}$. The left hand of $(6)$ is as 
follows:

$$
\begin{array}{r}
\sum_{\sigma \in X_{i j}, \tau \in X_{\ell k}}\left(\prod_{p=1}^{n-2} z_{p \sigma(p)} z_{p \tau(p)}\right)\left|\begin{array}{c}
e_{i} \\
e_{j} \\
e_{\sigma(1)} \\
\vdots \\
e_{\sigma(n-2)}
\end{array}\right|\left|\begin{array}{c}
e_{\ell} \\
e_{k} \\
e_{\tau(1)} \\
\vdots \\
e_{\tau(n-2)}
\end{array}\right| \\
+\sum_{\sigma \in X_{j k}, \tau \in X_{\ell i}}\left(\prod_{p=1}^{n-2} z_{p \sigma(p)} z_{p \tau(p)}\right)\left|\begin{array}{c}
e_{j} \\
e_{k} \\
e_{\sigma(1)} \\
\vdots \\
e_{\sigma(n-2)}
\end{array}\right|\left|\begin{array}{c}
e_{\ell} \\
e_{i} \\
e_{\tau(1)} \\
\vdots \\
e_{\tau(n-2)}
\end{array}\right| \\
+\sum_{\sigma \in X_{k i}, \tau \in X_{\ell j}}\left(\prod_{p=1}^{n-2} z_{p \sigma(p)} z_{p \tau(p)}\right)\left|\begin{array}{c}
e_{k} \\
e_{i} \\
e_{\sigma(1)} \\
e_{j} \\
e_{\tau(1)} \\
e_{\sigma(n-2)}
\end{array}\right|\left|\begin{array}{c}
\mid \\
e_{\tau(n-2)}
\end{array}\right|
\end{array}
$$

Let $(\sigma, \tau) \in X_{i j} \times X_{\ell k}$. We will find a unique $(\mu, \nu) \in X_{j k} \times X_{\ell i}$ (or $\left.(\mu, \nu) \in X_{k i} \times X_{\ell j}\right)$ such that $z_{p \sigma(p)} z_{p \tau(p)}=z_{p \mu(p)} z_{p \nu(p)}$ for each $p=$ $1, \cdots, n-2$ and that $\left|\begin{array}{c|c}e_{j} \\ e_{k} \\ e_{\mu(1)} \\ \vdots \\ e_{\mu(n-2)}\end{array}\right|\left|\begin{array}{c}e_{\ell} \\ e_{i} \\ e_{\nu(1)} \\ \vdots \\ e_{\nu(n-2)}\end{array}\right|$ is nonzero. (If $(\mu, \nu) \in X_{k i} \times X_{\ell j}$ then $\left|\begin{array}{c||c}e_{k} \\ e_{i} \\ e_{\mu(1)} \\ \vdots \\ e_{\mu(n-2)}\end{array}\right| \begin{gathered}e_{\ell} \\ e_{j} \\ e_{\nu(1)} \\ \vdots \\ e_{\nu(n-2)}\end{gathered} \mid$ is nonzero.) There exists a unique $p_{1}$ such that $k=\sigma\left(p_{1}\right)$, and then choose $\tau\left(p_{1}\right)$. If $\tau\left(p_{1}\right) \neq i$ and $\tau\left(p_{1}\right) \neq j$ then there exists a unique $p_{2}$ such that $\tau\left(p_{1}\right)=\sigma\left(p_{2}\right)$. If $\tau\left(p_{2}\right) \neq i$ and $\tau\left(p_{2}\right) \neq j$ then choose $p_{3}$ such that $\tau\left(p_{2}\right)=\sigma\left(p_{3}\right)$. This process stops only when $\tau\left(p_{r}\right)=i$ or $\tau\left(p_{r}\right)=j$, say $\tau\left(p_{r}\right)=i$, since there does not exist $p$ such that $\sigma(p)=i$ or $j$. Hence we get a unique sequence

$$
k=\sigma\left(p_{1}\right), \tau\left(p_{1}\right)=\sigma\left(p_{2}\right), \tau\left(p_{2}\right)=\sigma\left(p_{3}\right), \cdots, \tau\left(p_{r}\right)=i .
$$


Note that all terms of the sequence are different since all $\sigma\left(p_{1}\right), \cdots, \sigma\left(p_{r}\right), i$ are distinct. Set

$$
\begin{aligned}
& \mu(q)=\left\{\begin{array}{ll}
\sigma(q) & q \neq p_{m} \text { for all } m=1, \cdots, r \\
\tau\left(p_{m}\right) & q=p_{m}
\end{array},\right. \\
& \nu(q)= \begin{cases}\tau(q) & q \neq p_{m} \text { for all } m=1, \cdots, r \\
\sigma\left(p_{m}\right) & q=p_{m} .\end{cases}
\end{aligned}
$$

Then $\mu \in X_{j k}$ and $\nu \in X_{\ell i}$ and it is easy to see that $z_{p \sigma(p)} z_{p \tau(p)}=$ $z_{p \mu(p)} z_{p \nu(p)}$ for each $p=1, \cdots, n-2$ and each row of the matrices $\left(\begin{array}{c}e_{j} \\ e_{k} \\ e_{\mu(1)} \\ \vdots \\ e_{\mu(n-2)}\end{array}\right)$ and $\left(\begin{array}{c}e_{\ell} \\ e_{i} \\ e_{\nu(1)} \\ \vdots \\ e_{\nu(n-2)}\end{array}\right)$ is different, as claimed. Changing suitable rows in the matrices

$\left(\begin{array}{c}e_{j} \\ e_{k} \\ e_{\mu(1)} \\ \vdots \\ e_{\mu(n-2)}\end{array}\right)=\left(\begin{array}{c}e_{j} \\ e_{k} \\ e_{\sigma(1)} \\ \vdots \\ e_{\tau\left(p_{1}\right)}=e_{\sigma\left(p_{2}\right)} \\ \vdots \\ e_{\tau\left(p_{m}\right)}=e_{\sigma\left(p_{m+1}\right)} \\ \vdots \\ e_{\sigma(n-2)}\end{array}\right)$ and $\left(\begin{array}{c}e_{\ell} \\ e_{i} \\ e_{\nu(1)} \\ \vdots \\ e_{\nu(n-2)}\end{array}\right)=\left(\begin{array}{c}e_{\ell} \\ e_{i} \\ e_{\tau(1)} \\ \vdots \\ e_{\sigma\left(p_{1}\right)}=e_{k} \\ \vdots \\ e_{\sigma\left(p_{m}\right)}=e_{\tau\left(p_{m-1}\right)} \\ \vdots \\ e_{\tau(n-2)}\end{array}\right)$

we have $\left|\begin{array}{c}e_{j} \\ e_{k} \\ e_{\mu(1)} \\ \vdots \\ e_{\mu(n-2)}\end{array}\right|=(-1)^{r+1}\left|\begin{array}{c}e_{i} \\ e_{j} \\ e_{\sigma(1)} \\ \vdots \\ e_{\sigma(n-2)}\end{array}\right|$ and $\left|\begin{array}{c}e_{\ell} \\ e_{i} \\ e_{\nu(1)} \\ \vdots \\ e_{\nu(n-2)}\end{array}\right|=(-1)^{r}\left|\begin{array}{c}e_{\ell} \\ e_{k} \\ e_{\tau(1)} \\ \vdots \\ e_{\tau(n-2)}\end{array}\right|$,

hence

$$
\left|\begin{array}{c|c}
e_{i} \\
e_{j} \\
e_{\sigma(1)} \\
\vdots \\
e_{\sigma(n-2)}
\end{array}\right|\left|\begin{array}{c}
e_{\ell} \\
e_{k} \\
e_{\tau(1)} \\
\vdots \\
e_{\tau(n-2)}
\end{array}\right|=-\left|\begin{array}{c}
e_{j} \\
e_{k} \\
e_{\mu(1)} \\
\vdots \\
e_{\mu(n-2)}
\end{array}\right| \begin{gathered}
e_{\ell} \\
e_{i} \\
e_{\nu(1)} \\
\vdots \\
e_{\nu(n-2)}
\end{gathered} \mid
$$

Therefore (6) holds. 
Lemma 7. For any $1 \leq i, j, k \leq n, J_{\mathcal{F}}\left(x_{i}, x_{j}, x_{k}\right)=0$ if and only if

$$
\sum_{\ell=1}^{n}\left[\mathcal{F}_{i j} \frac{\partial \mathcal{F}_{\ell k}}{\partial x_{\ell}}+\mathcal{F}_{j k} \frac{\partial \mathcal{F}_{\ell i}}{\partial x_{\ell}}+\mathcal{F}_{k i} \frac{\partial \mathcal{F}_{\ell j}}{\partial x_{\ell}}\right]=0 .
$$

Proof. Since

$$
\begin{aligned}
\frac{\partial \mathcal{F}_{i j}}{\partial x_{\ell}} \mathcal{F}_{\ell k}+\frac{\partial \mathcal{F}_{j k}}{\partial x_{\ell}} \mathcal{F}_{\ell i}+\frac{\partial \mathcal{F}_{k i}}{\partial x_{\ell}} \mathcal{F}_{\ell j}= & \frac{\partial}{\partial x_{\ell}}\left(\mathcal{F}_{i j} F_{\ell k}+\mathcal{F}_{j k} \mathcal{F}_{\ell i}+\mathcal{F}_{k i} \mathcal{F}_{\ell j}\right) \\
- & {\left[\mathcal{F}_{i j} \frac{\partial \mathcal{F}_{\ell k}}{\partial x_{\ell}}+\mathcal{F}_{j k} \frac{\partial \mathcal{F}_{\ell i}}{\partial x_{\ell}}+\mathcal{F}_{k i} \frac{\partial \mathcal{F}_{\ell j}}{\partial x_{\ell}}\right], }
\end{aligned}
$$

(7) follows from (5) and (6).

Lemma 8. For any $\varphi^{1}, \cdots, \varphi^{n-2} \in B$, let $\mathcal{F}=\left(\begin{array}{c}\nabla \varphi^{1} \\ \vdots \\ \nabla \varphi^{n-2}\end{array}\right)$. Then $\sum_{\ell=1}^{n} \frac{\partial \mathcal{F}_{i \ell}}{\partial x_{\ell}}=0$ for each $i=1, \cdots, n$.

Proof. We may assume that $i \neq \ell$ since $\mathcal{F}_{i i}=0$. Denote by $X_{i \ell}$ the set of all bijective maps from $\{1, \cdots, n-2\}$ onto $\{1, \cdots, n\} \backslash\{i, \ell\}$ as in the proof of Lemma 6 and set

$$
<i, \ell>= \begin{cases}i+\ell-1 & \text { if } i<\ell, \\ i+\ell & \text { if } i>\ell .\end{cases}
$$

Then we have

$$
\begin{gathered}
\sum_{\ell=1, \ell \neq i}^{n} \frac{\partial \mathcal{F}_{i \ell}}{\partial x_{\ell}} \\
=\sum_{\ell=1, \ell \neq i}^{n} \sum_{\sigma \in X_{i \ell}}(-1)^{<i, \ell>} \operatorname{sgn}(\sigma) \frac{\partial}{\partial x_{\ell}}\left(\frac{\partial \varphi^{1}}{\partial x_{\sigma(1)}} \frac{\partial \varphi^{2}}{\partial x_{\sigma(2)}} \cdots \frac{\partial \varphi^{n-2}}{\partial x_{\sigma(n-2)}}\right) \\
=\sum_{\ell=1, \ell \neq i}^{n} \sum_{\sigma \in X_{i \ell}}(-1)^{<i, \ell>} \operatorname{sgn}(\sigma)\left[\frac{\partial^{2} \varphi^{1}}{\partial x_{\ell} \partial x_{\sigma(1)}}\left(\frac{\partial \varphi^{2}}{\partial x_{\sigma(2)}} \cdots \frac{\partial \varphi^{n-2}}{\partial x_{\sigma(n-2)}}\right)+\cdots\right. \\
\left.+\left(\frac{\partial \varphi^{1}}{\partial x_{\sigma(1)}} \cdots \frac{\partial \varphi^{n-3}}{\partial x_{\sigma(n-3)}}\right) \frac{\partial^{2} \varphi^{n-2}}{\partial x_{\ell} \partial x_{\sigma(n-2)}}\right]
\end{gathered}
$$

Fix a term

$$
A=\left(\frac{\partial \varphi^{1}}{\partial x_{\sigma(1)}} \cdots \frac{\partial \varphi^{k-1}}{\partial x_{\sigma(k-1)}}\right) \frac{\partial^{2} \varphi^{k}}{\partial x_{\ell} \partial x_{\sigma(k)}}\left(\frac{\partial \varphi^{k+1}}{\partial x_{\sigma(k+1)}} \cdots \frac{\partial \varphi^{n-2}}{\partial x_{\sigma(n-2)}}\right) .
$$


Let $\sigma(k)=j$. Define $\tau \in X_{i j}$ by $\tau(k)=\ell, \tau(q)=\sigma(q)$ for all $q \neq k$. Then the term

$$
B=\left(\frac{\partial \varphi^{1}}{\partial x_{\tau(1)}} \cdots \frac{\partial \varphi^{k-1}}{\partial x_{\tau(k-1)}}\right) \frac{\partial^{2} \varphi^{k}}{\partial x_{j} \partial x_{\tau(k)}}\left(\frac{\partial \varphi^{k+1}}{\partial x_{\tau(k+1)}} \cdots \frac{\partial \varphi^{n-2}}{\partial x_{\tau(n-2)}}\right)
$$

is equal to $A$ since $\frac{\partial^{2} \varphi^{k}}{\partial x_{\ell} \partial x_{j}}=\frac{\partial^{2} \varphi^{k}}{\partial x_{j} \partial x_{\ell}}$ for all $\ell, j, k$, and the coefficients of $A$ and $B$ are $(-1)^{<i, \ell>} \operatorname{sgn}(\sigma)$ and $(-1)^{<i, \ell>} \operatorname{sgn}(\tau)$, respectively. Let $|\ell-j|=m$. We may assume that $j<\ell<i$. Thus $\ell=j+m$. Let $\sigma\left(k_{1}\right)=\sigma(k)=j, \sigma\left(k_{2}\right)=j+1, \cdots, \sigma\left(k_{m}\right)=j+m-1$. Hence $\tau\left(k_{1}\right)=\ell, \tau\left(k_{2}\right)=j+1, \cdots, \tau\left(k_{m}\right)=j+m-1$. Arrange elements of $X_{i \ell}$ and $X_{i j}$ by using order relation:

$$
\begin{aligned}
& X_{i \ell}=\left\{p_{1}<p_{2}<\cdots<p_{n-2}\right\} \\
& =\{\cdots, j-1, \quad j, \cdots, \quad j+m-1=\ell-1, \quad \ell+1, \cdots\} \\
& X_{i j}=\left\{q_{1}<q_{2}<\cdots<q_{n-2}\right\} \\
& =\{\cdots, j-1, j+1, \cdots \text {, } \\
& \ell, \quad \ell+1, \cdots\} \text {. }
\end{aligned}
$$

Identifying $p_{s}$ and $q_{s}$ to $s$ for all $s=1, \cdots, n-2, \sigma$ and $\tau$ are permutations in $\{1, \cdots, n-2\}$ and $\tau^{-1} \sigma$ is defined as follows:

$$
\begin{gathered}
\tau^{-1} \sigma\left(k_{1}\right)=k_{2}, \tau^{-1} \sigma\left(k_{2}\right)=k_{3}, \tau^{-1} \sigma\left(k_{3}\right)=k_{4}, \cdots, \tau^{-1} \sigma\left(k_{m}\right)=k_{1} \\
\tau^{-1} \sigma(p)=p \text { for all } p \neq k_{q}, q=1, \cdots, m .
\end{gathered}
$$

Thus $\tau^{-1} \sigma$ is the cycle $\left(k_{1} k_{2} \cdots k_{m}\right)$ in the set $\{1, \cdots, n-2\}$. Hence $\operatorname{sgn}(\sigma)=\operatorname{sgn}(\tau)$ if and only if $\ell-j=m$ is odd $\operatorname{since} \operatorname{sgn}(\tau)=\operatorname{sgn}\left(\tau^{-1}\right)$. It follows that the coefficient $(-1)^{<i, \ell>} \operatorname{sgn}(\sigma)$ of $A$ is $-(-1)^{<i, j>} \operatorname{sgn}(\tau)$ and thus $\sum_{\ell=1, \ell \neq i}^{n} \frac{\partial \mathcal{F}_{i \ell}}{\partial x_{\ell}}=0$.

Theorem 9. For any $\varphi^{1}, \cdots, \varphi^{n-2} \in B$, let $\mathcal{F}=\left(\begin{array}{c}\nabla \varphi^{1} \\ \vdots \\ \nabla \varphi^{n-2}\end{array}\right)$. Then $B$ is a Poisson algebra under $\{\cdot, \cdot\}^{\mathcal{F}}$.

Proof. It is enough to show that $J_{\mathcal{F}}\left(x_{i}, x_{j}, x_{k}\right)=0$ for all $1 \leq i, j, k \leq$ $n$ by Proposition 4 . By Lemma 8, (7) holds since $\mathcal{F}_{i \ell}=-\mathcal{F}_{\ell i}$, and thus we have $J_{\mathcal{F}}\left(x_{i}, x_{j}, x_{k}\right)=0$ for all $1 \leq i, j, k \leq n$ by Lemma 7 .

Lemma 10. Let $\mathcal{F}$ and $\mathcal{G}$ be $(n-2) \times n$-matrices in $B$ such that $\mathcal{G}_{i}=a_{i} \mathcal{F}_{i}$ for each $i$, where $a_{i} \in B$ and $\mathcal{F}_{i}$ and $\mathcal{G}_{i}$ are the $i$-rows of $\mathcal{F}$ and $\mathcal{G}$ respectively. If $\{\cdot, \cdot\}^{\mathcal{F}}$ is a Poisson bracket on $B$ then $\{\cdot, \cdot\}^{\mathcal{G}}$ is also a Poisson bracket and $\{\cdot, \cdot\}^{\mathcal{G}}=\left(a_{1} \cdots a_{n-2}\right)\{\cdot, \cdot\}^{\mathcal{F}}$. 
Proof. Set $a=a_{1} \cdots a_{n-2}$. Since $\mathcal{G}_{i j}=a \mathcal{F}_{i j}$ for all $i, j$, we have

$$
\begin{aligned}
\frac{\partial \mathcal{G}_{i j}}{\partial x_{\ell}} \mathcal{G}_{\ell k}+\frac{\partial \mathcal{G}_{j k}}{\partial x_{\ell}} \mathcal{G}_{\ell i}+\frac{\partial \mathcal{G}_{k i}}{\partial x_{\ell}} \mathcal{G}_{\ell j}=a^{2} & {\left[\frac{\partial \mathcal{F}_{i j}}{\partial x_{\ell}} \mathcal{F}_{\ell k}+\frac{\partial \mathcal{F}_{j k}}{\partial x_{\ell}} \mathcal{F}_{\ell i}+\frac{\partial \mathcal{F}_{k i}}{\partial x_{\ell}} \mathcal{F}_{\ell j}\right] } \\
& +a \frac{\partial a}{\partial x_{\ell}}\left(\mathcal{F}_{i j} F_{\ell k}+\mathcal{F}_{j k} \mathcal{F}_{\ell i}+\mathcal{F}_{k i} \mathcal{F}_{\ell j}\right) .
\end{aligned}
$$

Thus $\{\cdot, \cdot\}^{\mathcal{G}}$ is a Poisson bracket by (5) and (6).

Corollary 11. Fix $s_{1}, t_{1}, \cdots, s_{n-2}, t_{n-2} \in A$ such that $s_{i}$ and $t_{i} \neq 0$ are coprime for each $i=1,2, \cdots, n-2$. Then (3) is a Poisson bracket on $A$.

Proof. Under the notation of Theorem 9 and Lemma 10, set $\varphi^{i}=s_{i} / t_{i}$ and $a_{i}=t_{i}^{2}$ for all $i=1, \cdots, n-2$. Then the result follows immediately by Theorem 9 and Lemma 10 since each component of $t_{i}^{2} \nabla\left(s_{i} / t_{i}\right)$ is an element of $A$.

\section{References}

[1] D. A. Jordan and S. Oh, Poisson brackets and Poisson spectra in polynomial algebras, New Trends in Noncommutative Algebra, Contemp. Math. 562 (2012), 169-187.

[2] _ Poisson spectra in polynomial algebras, arXiv:math.RA/1212.5158 (2012).

[3] A. N. Panov, n-Poisson and n-Sklyanin brackets, Journal of Mathematical Sciences 110 (2002), 2322-2329.

[4] R. Przybysza, On one class of exact Poisson structures, Journal of Mathematical Physics 424 (2001), no. 4, 1913-1920. 
Department of Mathematics

Chungnam National University

Daejeon 305-764, Republic of Korea

E-mail: jhahn@cnu.ac.kr

$* *$

Department of Mathematics

Chungnam University

Daejeon 305-764, Republic of Korea

E-mail: sqoh@cnu.ac.kr

$* * *$

Department of Mathematics

Chungnam University

Daejeon 305-764, Republic of Korea 